\title{
Novel Sliding Mode Control for Ball Screw Servo System
}

\author{
Rongrong Qian ${ }^{1}$, Minzhou $\mathrm{Luo}^{2}$, Jianghai Zhao ${ }^{2}$ and $\mathrm{Tao}_{\mathrm{Li}}{ }^{2}$ \\ ${ }^{1}$ School of Information Science and Technology, University of Science and Technology of China, Hefei, Anhui, China \\ ${ }^{2}$ Institute of Advanced Manufacturing Technology, Hefei Institute of Physical Science, Chinese Academy of Sciences, Changzhou, Jiangsu, \\ China
}

\begin{abstract}
This paper presents a novel sliding mode control to achieve a high positioning accuracy for ball screw servo system. A two-degree-of-freedom (2-DOF) model with time-varying parameter uncertainties and disturbance is firstly built to describe the first axial mode resonance for the ball screw servo system. A novel nonlinear sliding mode control is designed for the system with better transient response of fast response and non-overshoot. The stability of the nonlinear sliding-mode surface and controller are verified based on the Lyapunov function. The frequency response experiment result indicates that the system modelling is accurate enough and the simulation result shows that the tracking accuracy for ball screw servo system is improved with the proposed controller. Keywords. ball screw servo system; sliding mode control; nonlinear sliding-mode surface; modeling
\end{abstract}

\section{Introduction}

Ball screw servo system is widely used in industrial high positioning facilities due to its high transmission stability and low sensitivity to external forces and inertia variations [1]. Since the high accuracy with high speed and high acceleration are the main demands and developing trends, it is particularly important to design the superior control law on the trajectory tracking for ball screw servo system.

As for the topic of tracking control system design, a large number of control strategies emerge to acquire the high performance tracking accuracy [2-5]. However, sliding mode control is employed frequently in the tracking control. For example, integral sliding mode controller [6] and adaptive sliding mode controller [7, 8] are presented to achieve high-precision positioning performance with repeatability and robustness. However, in order to improve the system convergence characteristics and transient response performance, Bijnan Bandyopadhyay et al. present a novel nonlinear sliding surface according to the composite nonlinear feedback principle [9]. With this nonlinear sliding-mode surface, the system can have a small damping ratio with fast response and along with the system getting close to the desired trajectory the damping ratio is getting bigger to avoid the overshoot. Apparently, it has the advantages of settling quickly without overshoot comparing to linear sliding mode controller.

In this paper, a control scheme is proposed to improve the sliding mode control based on the nonlinear slidingmode surface for the ball screw servo system. The remainder of this paper is organized as follows. In section
2, the structure of the ball screw servo system is given. A two-degree-of-freedom (2-DOF) model describing the first axial mode resonance which has the most influence for the positioning accuracy in the ball screw servo system is built. In section 3, a novel sliding mode controller (NSMC) based on nonlinear sliding mode control is presented and derived for the 2-DOF model of ball screw servo system with bounded parameter uncertainties and external disturbances. The stability of the nonlinear sliding-mode surface and the NSMC are verified according to the Lyapunov function. In the end, the frequency response experiment is conducted to identify the 2-DOF model for ball screw servo system and the controller simulation of NSMC compared with traditional linear sliding mode control for the system is given.

\section{Two-DOF system modeling}

The structure of ball screw servo system analyzed in this paper is shown in Figure 1. It consists of ball screw driven by an $\mathrm{AC}$ motor through a coupling and assembled with the machine base by deep groove ball bearings. The worktable is mounted to the ball nut and constrained to the bilateral linear guides in order to move axially. 


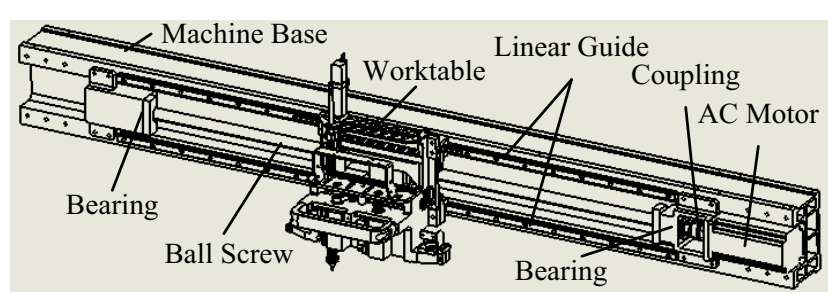

Figure 1. The structure of ball screw servo system

In the ball screw servo system, the first axial mode resonance has the most impact on the worktable linear positioning accuracy. The flexible characteristic of the translating components is the main reason for the first axial mode resonance. It includes the inherent flexibility of the ball screw and bearings and the elastic interaction characteristics between the worktable, nut and ball screw. A simplified model of two mass drive system shown in Figure 2 is used to simulate this flexible characteristic to capture the first axial dynamic for ball screw servo system and the 2-DOF mathematical model of the system is expressed as Eq. (1),

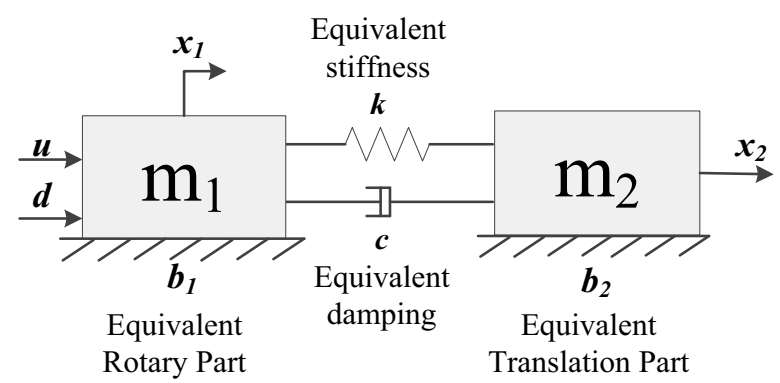

Figure 2. Simplified model for ball screw servo system

$$
\begin{aligned}
& m_{1} \ddot{x}_{1}=-b_{1} \dot{x}_{1}+k\left(x_{2}-x_{1}\right)+c\left(\dot{x}_{2}-\dot{x}_{1}\right)+u(t)+d(t) \\
& m_{2} \ddot{x}_{2}=-b_{2} \dot{x}_{2}+k\left(x_{1}-x_{2}\right)+c\left(\dot{x}_{1}-\dot{x}_{2}\right)
\end{aligned}
$$

Where $m_{1}$ is the rotary inertia equivalent to the motor shaft and $m_{2}$ is the equivalent inertia of worktable. $b_{1}$ is the viscous friction between the motor shaft and rotary bearing. $b_{2}$ is the viscous friction of the bilateral guides. $k$ represents the total equivalent axial stiffness and $c$ represents the damping in the preloaded nut. $u(t)$ is the control input voltage used to generate the motor torque to actuate the ball screw platform. $x_{1}(t)$ is the equivalent rotary displacement of the motor shaft and $x_{2}(t)$ is the equivalent rotary displacements of worktable multiplied by the screw pitch. $d(t)$ represents the bounded parameter uncertainties and equivalent external disturbances of the system. In this paper, $d(t)$ is regarded as the matched disturbance to the input.

The transfer function of 2-DOF model and the relation between $x_{1}(t)$ and $x_{2}(t)$ are derived from (1) as follows,

$$
\begin{aligned}
& G_{1}(s)=\frac{x_{1}(s)}{u(s)}=\frac{m_{2} s^{2}+\left(b_{2}+c\right) s+k}{a_{4} s^{4}+a_{3} s^{3}+a_{2} s^{2}+a_{1} s} \\
& G_{2}(s)=\frac{x_{2}(s)}{u(s)}=\frac{c s+k}{a_{4} s^{4}+a_{3} s^{3}+a_{2} s^{2}+a_{1} s} \\
& \frac{x_{2}(s)}{x_{1}(s)}=\frac{c s+k}{m_{2} s^{2}+\left(b_{2}+c\right) s+k}
\end{aligned}
$$

Where $a_{4}=m_{1} m_{2}, a_{3}=m_{1}\left(b_{2}+c\right)+m_{2}\left(b_{1}+c\right), a_{2}=$ $k\left(m_{1}+m_{2}\right)+b_{1} b_{2}+b_{1} c+b_{2} c, a_{1}=k\left(b_{1}+b_{2}\right)$.

The transfer functions (2)-(4) are used to identify the 2-DOF model parameters according to the frequency response measuring from the linear encoder which is to be discussed later.

The state space model transformed from Eq. (1) with the state vector $x(t)=\left[x_{2}(t), x_{1}(t), \dot{x}_{2}(t), \dot{x}_{1}(t)\right]^{\mathrm{T}}$ is inferred as follows,

$$
\begin{aligned}
& {\left[\begin{array}{c}
\dot{x}_{2}(t) \\
\dot{x}_{1}(t) \\
\ddot{x}_{2}(t) \\
\ddot{x}_{1}(t)
\end{array}\right]=\left[\begin{array}{cccc}
0 & 0 & 1 & 0 \\
0 & 0 & 0 & 1 \\
-\frac{k}{m_{2}} & \frac{k}{m_{2}} & -\frac{b_{2}+c}{m_{2}} & \frac{c}{m_{2}} \\
\frac{k}{m_{1}} & -\frac{k}{m_{1}} & \frac{c}{m_{1}} & -\frac{b_{1}+c}{m_{1}}
\end{array}\right]\left[\begin{array}{c}
x_{2}(t) \\
x_{1}(t) \\
\dot{x}_{2}(t) \\
\dot{x}_{1}(t)
\end{array}\right]+} \\
& {\left[\begin{array}{c}
0 \\
0 \\
0 \\
\frac{1}{m_{1}}
\end{array}\right] u(t)+\left[\begin{array}{c}
0 \\
0 \\
0 \\
\frac{1}{m_{1}}
\end{array}\right] d(t) }
\end{aligned}
$$

The state vector is divided into two parts as $z_{1}(t)=\left[x_{2}(t), x_{1}(t), \dot{x}_{2}(t)\right]^{\mathrm{T}} \quad$ and $\quad z_{2}(t)=\dot{x}_{1}(t) \quad$. The corresponding state space model is,

$$
\left[\begin{array}{l}
\dot{z}_{1}(t) \\
\dot{z}_{2}(t)
\end{array}\right]=\left[\begin{array}{ll}
A_{11} & A_{12} \\
A_{21} & A_{22}
\end{array}\right]\left[\begin{array}{l}
z_{1}(t) \\
z_{2}(t)
\end{array}\right]+\left[\begin{array}{l}
\mathbf{0} \\
B
\end{array}\right] u(t)+\left[\begin{array}{l}
\mathbf{0} \\
B
\end{array}\right] d(t)
$$

Where,

$$
\begin{gathered}
A_{11}=\left[\begin{array}{ccc}
0 & 0 & 1 \\
0 & 0 & 0 \\
-k / m_{2} & k / m_{2} & -b_{2}-c
\end{array}\right], A_{12}=\left[\begin{array}{c}
0 \\
1 \\
c / m_{2}
\end{array}\right], \\
A_{21}=\left[\begin{array}{lll}
\frac{k}{m_{1}} & -\frac{k}{m_{1}} & \frac{c}{m_{1}}
\end{array}\right], A_{22}=-\frac{b_{1}+c}{m_{1}}, A=\left[\begin{array}{ll}
A_{11} & A_{12} \\
A_{21} & A_{22}
\end{array}\right], \\
B=\frac{1}{m_{1}} .
\end{gathered}
$$

\section{Novel sliding mode control}

The novel sliding mode control (NSMC) based on nonlinear sliding-mode surface is presented here to suppress the first axial resonance of the ball screw servo system with bounded disturbance and parameter uncertainties. The design and stability verification of the control strategy are derived based on the Lyapunov function.

\subsection{Nonlinear sliding-mode surface and stability analysis}

The desired state trajectory for system (6) is defined as,

$$
\begin{aligned}
& z_{\text {ref }}=\left[\begin{array}{ll}
z_{1 r e f} & z_{2 r e f}
\end{array}\right]^{\mathrm{T}} \\
& z_{1 r e f}(t)=\left[\begin{array}{lll}
x_{2 r e f}(t) & x_{1 r e f}(t) & \dot{x}_{2 r e f}(t)
\end{array}\right]^{\mathrm{T}} \\
& z_{2 r e f}(t)=\dot{x}_{1 r e f}(t)
\end{aligned}
$$


In this paper, the continuous acceleration reference trajectory is used as $x_{2 \text { ref. }} x_{1 r e f}$ is acquired from equation (4) with the nominal parameters.

The system state errors are given as,

$$
\begin{aligned}
& e_{1}(t)=z_{1}(t)-z_{1 r e f}(t) \\
& e_{2}(t)=z_{2}(t)-z_{2 r e f}(t)
\end{aligned}
$$

The nonlinear sliding-mode surface $s(z, t)$ for the system is defined as,

$$
s(z, t)=\sigma(y, r) z(t)=\left[\begin{array}{lll}
F-\psi(y, r) A_{12}^{\mathrm{T}} P & 1
\end{array}\right]\left[\begin{array}{l}
e_{1}(t) \\
e_{2}(t)
\end{array}\right]
$$

Where $\sigma(y, r)=\left[F-\psi(y, r) A_{12}^{\mathrm{T}} P \quad 1\right]$ and $r$ is the desired worktable equivalent rotary displacement. $F$ is chosen to satisfy the condition that $A_{11}-A_{12} F$ has stable eigenvalues and the dominant poles have a very low damping ratio [9]. $P$ is the positive definite symmetric matrix satisfied the Lyapunov equation below,

$$
\left(A_{11}-A_{12} F\right)^{\mathrm{T}} P+P\left(A_{11}-A_{12} F\right)=-W
$$

Where $W$ is a known positive definite matrix.

$\psi(y, r)$ is a non-positive function and continuously differentiable with respect to $y$. The use of $\psi(y, r)$ is to increase the damping ratio of system by changing itself from 0 or a tiny value to a negative constant as the actual value is gradually moving closely to the expected value. In order to satisfy these requirements, $\psi(y, r)$ is chosen as follows,

$$
\psi(y, r)=-\beta\left(e^{-\rho(y-r)^{2}}\right)
$$

Where $\beta$ is used to adjust the damping ratio and satisfies $\beta>0$ and $\rho$ should be big enough to make $\psi(y, r)$ to be a tiny value initially.

On the basis of the control principle of sliding mode control, the system states will be forced to the slidingmode surface (11) by the control law in finite time and the following equation is satisfied,

$$
s(z, t)=0
$$

Combining equations (6), (10) and (11) gives that,

$$
\begin{gathered}
e_{2}(t)=-F e_{1}(t)+\psi(y, r) A_{12}^{\mathrm{T}} P e_{1}(t) \\
\dot{e}_{1}(t)=\left(A_{11}-A_{12} F+A_{12} \psi(y, r) A_{12}^{\mathrm{T}} P\right) e_{1}(t)
\end{gathered}
$$

Therefore,

$$
e_{1}(t)=0 \Rightarrow e_{2}(t)=0
$$

The following Lyapunov function is given to analyze the stability of the system states,

$$
V_{1}(t)=e_{1}^{\mathrm{T}}(t) P e_{1}(t) .
$$

Then,

$$
\begin{aligned}
\dot{V}_{1}(t)= & \dot{e}_{1}^{\mathrm{T}}(t) P e_{1}(t)+e_{1}^{\mathrm{T}}(t) P \dot{e}_{1}(t) \\
= & e_{1}^{\mathrm{T}}(t)\left(A_{11}-A_{12} F\right)^{\mathrm{T}} P e_{1}(t)+e_{1}^{\mathrm{T}}(t) P\left(A_{11}-A_{12} F\right) e_{1}(t)+ \\
& 2 e_{1}^{\mathrm{T}}(t) P A_{12} \psi(y, r) A_{12}^{\mathrm{T}} P e_{1}(t)<0
\end{aligned}
$$

From above, it can be seen that the error state $e_{1}(t)$ is asymptotic convergence as well as $e_{2}(t)$.

\subsection{NSMC design and stability analysis}

The NSMC is designed to drive the system (5) move to the nonlinear sliding-mode surface (11). The exponential approaching law (19) is combined here to design the controller.

$$
\dot{s}(z, t)=-k_{1} s(z, t)-k_{2} \operatorname{sgn}(s(z, t))
$$

Where $k_{1}>0$ and $k_{2}>0$.

The derivative of sliding-mode surface Eq. (11) is as follows,

$$
\begin{aligned}
\dot{s}(z, t) & =\left[\begin{array}{lll}
-\frac{d}{d t} \psi A_{12}^{\mathrm{T}} P & 0
\end{array}\right]\left[\begin{array}{c}
e_{1}(t) \\
e_{2}(t)
\end{array}\right]+\left[\begin{array}{ll}
F-\psi A_{12}^{\mathrm{T}} P & 1
\end{array}\right]\left[\begin{array}{c}
\dot{e}_{1}(t) \\
\dot{e}_{2}(t)
\end{array}\right] \\
& =-\frac{d}{d t} \psi A_{12}^{\mathrm{T}} P e_{1}(t)+\left(F-\psi A_{12}^{\mathrm{T}} P\right) \dot{e}_{1}(t)+\dot{e}_{2}(t) \\
& =-\frac{d}{d t} \psi A_{12}^{\mathrm{T}} P e_{1}(t)+\left(F-\psi A_{12}^{\mathrm{T}} P\right)\left(\dot{z}_{1}(t)-\dot{\dot{1}}_{1 r f}(t)\right)+\left(\dot{z}_{2}(t)-\dot{z}_{2 r f}(t)\right) \\
& =-\frac{d}{d t} \psi A_{12}^{\mathrm{T}} P e_{1}(t)+\sigma(t) A z(t)-\sigma(t) \dot{z}_{\text {ref }}+B u(t)+d(t)
\end{aligned}
$$

The controller could be designed as follows by combining with Eq.(19),

$$
\begin{aligned}
u(t) & =-B^{-1}\left(-\frac{d}{d t} \psi(y, r) A_{12}^{\mathrm{T}} P e_{1}(t)-\sigma(t) \dot{z}_{r e f}(t)\right. \\
& \left.+\sigma(t) A z(t)+k_{1} s+k_{2}(t) * \operatorname{sgn}(s)\right)
\end{aligned}
$$

Where $k_{2}$ should be chosen big enough to satisfy $k_{2} \geq|d(t)|$.

In order to analyze the stability of proposed controller, the Lyapunov candidate function is considered as follows,

$$
V_{2}(t)=\frac{1}{2} s^{2}(z, t)
$$

The derivative of equation (22) is,

$$
\begin{aligned}
\dot{V}_{2}(t) & =s(z, t) \dot{s}(z, t) \\
& =s(z, t) \cdot\left(-k_{1} s(z, t)-k_{2} \operatorname{sgn}(s(z, t))+d(t)\right) \\
& =-k_{1} s(z, t)^{2}-k_{2}|s(z, t)|+d(t) s(z, t) \\
& <-k_{1} s(z, t)^{2}-k_{2}|s(z, t)|+d(t)|s(z, t)| \\
& =-k_{1} s(z, t)^{2}+\left(d(t)-k_{2}\right)|s(z, t)| \\
& <0
\end{aligned}
$$

Therefore, the system state errors of Eq.(10) can be driven to the sliding-mode surface and converge to zero by the control law (21).

A saturation function shown in Eq.(23) with a certain threshold value is used here to instead of the sign function in equation (21) to weaken the chattering of the system,

$$
\operatorname{sat}(u)=\left\{\begin{array}{ll}
-1, & u<-\ell \\
\frac{1}{\ell}, & |u| \leq \ell . \\
1, & u>\ell
\end{array} .\right.
$$

\subsection{Advantage of system transient response by NSMC}

In order to make the point that the transient response of system controlled by NSMC is improved comparing with the traditional linear sliding mode controls (LSMCs), a step response of permanent magnet synchronous motor (PMSM) simulation system is used here to compare the three controllers of the LSMC with under-damping (LSMC-UD) and LSMC with over-damping (LSMC-OD) and NSMC. The step response results are shown in Figure 3 and it can be concluded evidently that NSMC control algorithm can acquire the excellent transient response performance with fast response and nonovershoot which is conducive to ball screw servo system. 


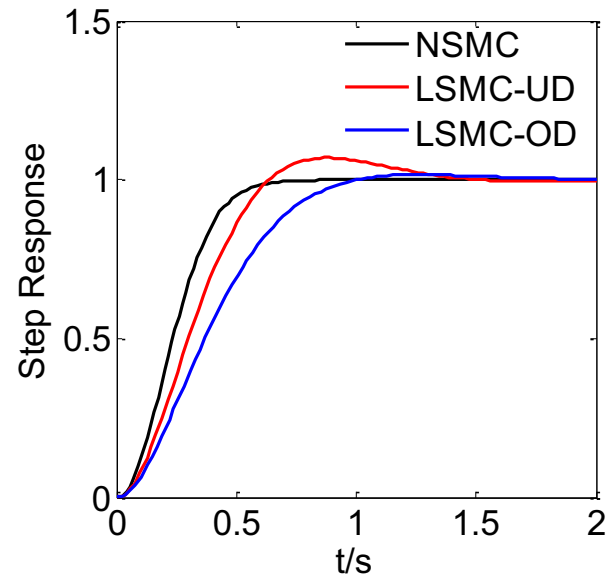

Figure 3. Step response for PMSM simulation system with three controllers

\section{Simulation and experiment results}

The ball screw servomechanism is shown in Figure 4. The worktable is supported by two THK SHS20C linear ball rolling guide-ways and driven by a THK precision ball screw of WGF2040-3G0+1170LC3 with 40mm pitch and $20 \mathrm{~mm}$ diameter. $1.3 \mathrm{~kW}$ Mitsubishi servo motor is used with the driver MR-J3-70A to actuate the ball-screw through a MISUMI diaphragm coupling CPDT40-12-19. This setup is applied in the surface mounting machine. The high-resolution rotary and linear encoders are used in the detection process. The rotary encoder is in the motor which is setting to generate 40000 sinusoidal signals per revolution. The Renishaw optical incremental linear encoder RGH22Q50D00A is used to detect the translational displacement of the worktable. Both encoders are setting to have the position measurement resolution of $1 \mu \mathrm{m}$. The frequency response experiment is implemented at $20 \mathrm{kHz}$ sampling frequency on dSPACE DS1104 system.

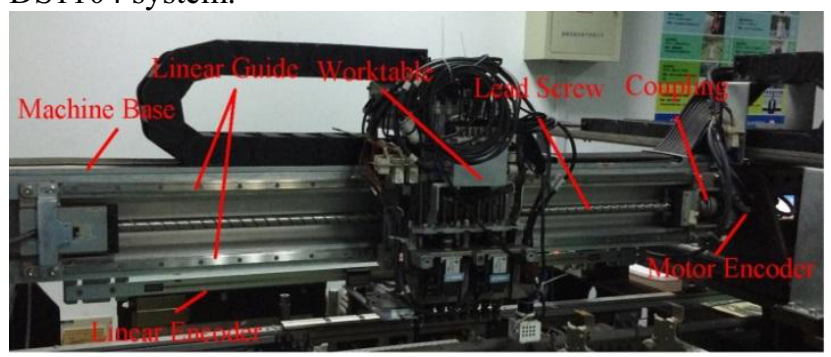

Figure 4. Ball screw drive servomechanism

\subsection{Frequency response experiment modeling}

In order to acquire the first axial mode resonance, the frequency response experiment is conducted by exciting the drive motor with a torque command which is a sinusoidal input voltage with amplitude $0.2 \mathrm{~V}$ at frequency ranges from $1 \mathrm{~Hz}$ to $400 \mathrm{~Hz}$ gradually increasing by $1 \mathrm{~Hz}$ increment. The 2-DOF model is obtained by the identification of Eq.(2)-(4) from the experiment result by the method of pole placement principle and least square method and shown as follows,

$$
\begin{gathered}
m_{1}=5.4741 \times 10^{-4}\left(\mathrm{~V} / \mathrm{rad} / \mathrm{s}^{2}\right), \\
m_{2}=10.0842 \times 10^{-4}\left(\mathrm{~V} / \mathrm{rad} / \mathrm{s}^{2}\right), \\
k=353.2717(\mathrm{~V} / \mathrm{rad}), c=0.020(\mathrm{~V} / \mathrm{rad} / \mathrm{s}), \\
b_{1}=0.0013(\mathrm{~V} / \mathrm{rad} / \mathrm{s}), b_{2}=0.0013(\mathrm{~V} / \mathrm{rad} / \mathrm{s})
\end{gathered}
$$

Figure 5 shows the comparison of frequency response between the 2-DOF model and experiment measurement. It can be seen that the frequency responses of model are in good agreement with the first axial resonant frequency of the test bed.
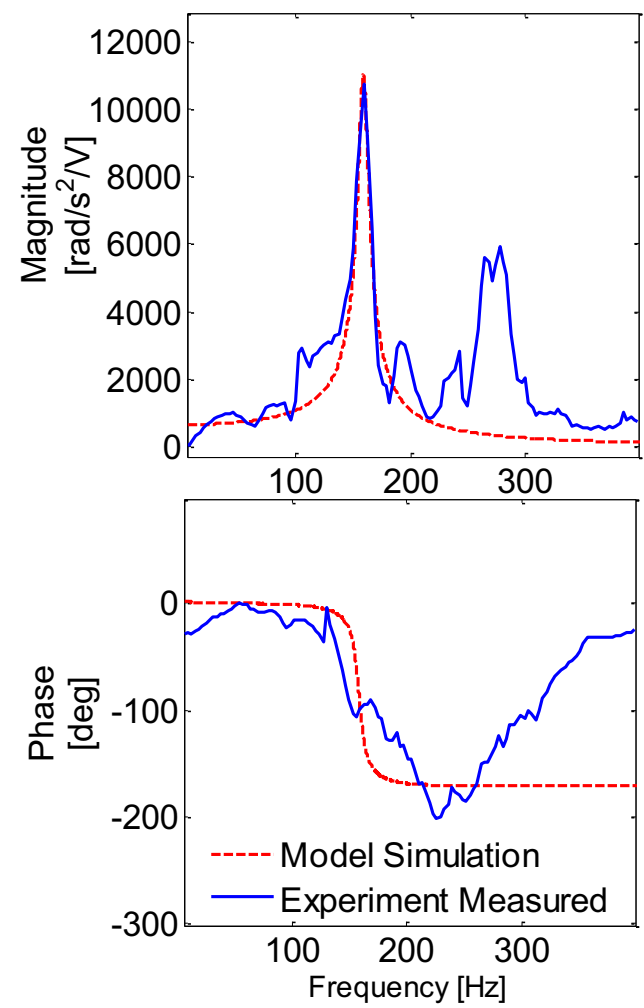

Figure 5. Frequency response comparison of model simulation and experiment measured

\subsection{Controller simulation}

In order to verify the effectiveness of the proposed NSMC in the ball screw servo system, two LSMC controllers with different damping ratios are used for comparison. The acceleration continuous signal shown in Figure 6 is used as reference trajectory here. The maximum acceleration of the reference is $10 \mathrm{~m} / \mathrm{s}^{2}$ and maximum velocity is $1.1 \mathrm{~m} / \mathrm{s}$. The equivalent motion of the worktable with the reference trajectory is a back and forth motion in the ball screw servo system.

The damping ratios of LSMC_1 and LSMC_ 2 are 0.5 and 0.99 respectively and these values are the initial and ultimate damping ratio in succession for NSMC controller.

The system parameter perturbations and disturbances in simulations are hypothesized as a whole as follows,

$$
d(t)=10 \sin (100 t)
$$

Figure 7 shows the tracking errors and control signals for the three controllers for ball screw servo system. It 
can be seen that the tracking error is reduced considerably by NSMC algorithm compared with the LSMCs. Meanwhile, the control voltage of the NSMC is decreased in somehow and this is beneficial for the power reduction.
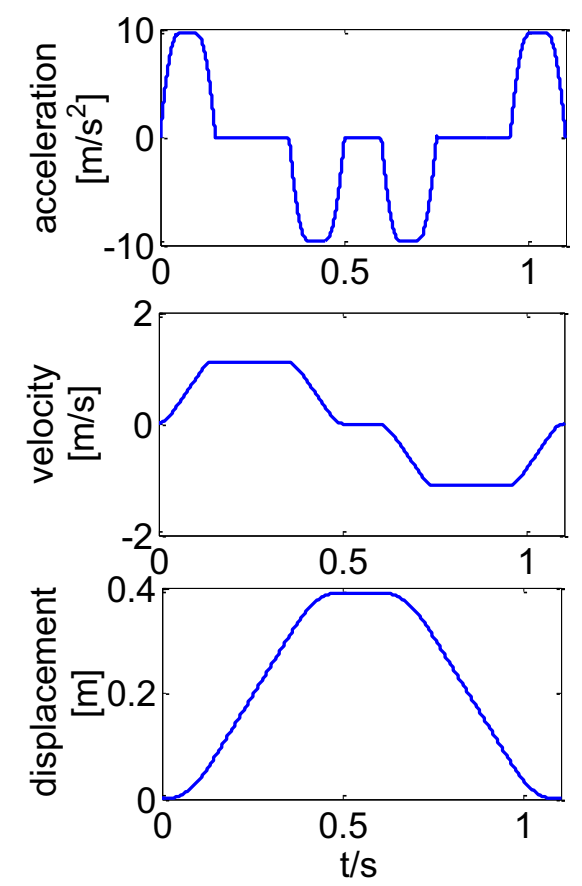

Figure 6. Reference Trajectory

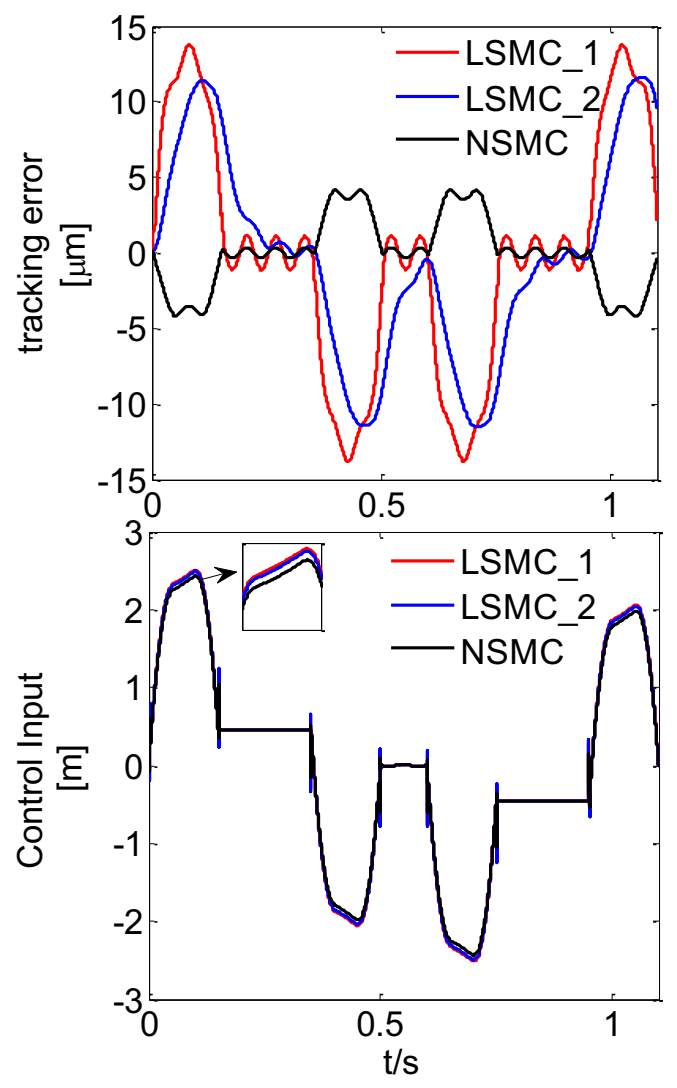

Figure 7. Three control strategies simulation comparison

\section{Conclusion}

The modeling and trajectory tracking control for a high speed ball screw servo system has been researched. A novel sliding mode controller based on nonlinear slidingmode surface is designed to take full advantages of high transient response performance with fast response and non-overshoot on the ball screw servo system. The experiment and simulation results show that the system modelling is accurate enough and the tracking accuracy of the system is improved appreciably by the proposed control strategy. The designed control strategies could be applied in more plants to improve the transient response of the systems.

\section{Acknowledgements}

This paper is supported by the Project of National Nature Science Foundation of China (No. 51405469) and the Project of National Science-Technology Support Plan of China (No. 2015BAK06B02).

\section{References}

1. D. A. Vicente, R. L. Hecker, F. J. Villegas, \& G. M. Flores, Modeling and vibration mode analysis of a ball screw drive. International Journal of Advanced Manufacture Technology, 58(1): 257-265 (2012)

2. H. Zhu \& H. Fujimoto, Mechanical deformation analysis and high-precision control for ball-screwdriven stages. IEEE/ASME Transactions on Mechatronics, 20(2): 956-966 (2015)

3. H. Fujimoto \& T. Takemura, High-precision control of ball-screw-driven stage based on repetitive control using-times learning filter. IEEE Transactions on Industrial Electronics, 61(7): 3694-3703 (2014)

4. T. Yamamoto, M. Naito, G. Chen, et al. Robust control design for ball screw system via descriptor representation and loop shaping. in IEEE 2014 Proceedings of the $6^{\text {th }}$ International Conference on Modeling Identification \& Control (ICMIC), 2014: 82-87 (2014)

5. M. Hanifzadegan and R. Nagamune, Tracking and structural vibration control of flexible ball-screw drives with dynamic variations. IEEE/ASME Transactions on Mechatronics, 20(1): 133-142 (2015)

6. C. L. Chen, M. J. Jang, and K. C. Lin, Modeling and high-precision control of a ball-screw-driven stage. Precision Engineering, 28(4), 483-495 (2004)

7. K. Erkorkmaz and A. Kamalzadeh, High bandwidth control of ball screw drives. CIRP Annals Manufacturing Technology, 55(1), 393-398 (2006)

8. L. Dong \& W. C. Tang, Adaptive backstepping sliding mode control of flexible ball screw drives with time-varying parametric uncertainties and disturbances. ISA Transactions, 53(1), 110-116 (2014)

9. B. Bandyopadhyay, D. Fulwani, and L. Fridman, Sliding mode control using novel sliding surface. Springer (2007). 